\title{
Improving Fuzzy Multilevel Graph Embedding through Feature Selection Technique
}

\author{
Muhammad Muzzamil Luqman ${ }^{1,2}$, Jean-Yves Ramel ${ }^{1}$, and Josep Lladós ${ }^{2}$ \\ 1 Laboratoire d'Informatique, Université François Rabelais de Tours, 37200 France \\ 2 Computer Vision Center, Universitat Autònòma de Barcelona, 08193 Spain \\ \{luqman,ramel\}@univ-tours.fr, josep@cvc.uab.es
}

\begin{abstract}
Graphs are the most powerful, expressive and convenient data structures but there is a lack of efficient computational tools and algorithms for processing them. The embedding of graphs into numeric vector spaces permits them to access the state-of-the-art computational efficient statistical models and tools. In this paper we take forward our work on explicit graph embedding and present an improvement to our earlier proposed method, named "fuzzy multilevel graph embedding FMGE", through feature selection technique. FMGE achieves the embedding of attributed graphs into low dimensional vector spaces by performing a multilevel analysis of graphs and extracting a set of global, structural and elementary level features. Feature selection permits FMGE to select the subset of most discriminating features and to discard the confusing ones for underlying graph dataset. Experimental results for graph classification experimentation on IAM letter, GREC and fingerprint graph databases, show improvement in the performance of FMGE.
\end{abstract}

Keywords: graphics recognition, graph classification, explicit graph embedding, feature selection.

\section{Introduction and Related Works}

Over decades of research in pattern recognition, the research community has developed a range of expressive and powerful approaches for diverse problem domains. Graph based structural representations are widely employed for extracting the structure, topology and geometry, in addition to the statistical details of underlying data 1. During next step in the processing chain, generally these representations could not be exploited to their full strength because of limited availability of computational tools for them. On the other hand, the efficient and mature computational models offered by statistical approaches, work only on vector data and cannot be directly applied to these high-dimensional representations. The emerging domain of graph embedding in pattern recognition, addresses this problem of the lack of efficient computational tools for graph based representations.

Graph embedding is a methodology aimed at representing a whole graph, along-with the attributes attached to its nodes and edges, as a point in a suitable 
vector space. Graph embedding is a natural outcome of parallel advancements in structural and statistical pattern recognition. It offers a straightforward solution, by employing the representational power of symbolic data structures and the computational superiority of feature vectors [2]. It acts as a bridge between structural and statistical approaches [3] 4], and allows a pattern recognition method to benefit from computational efficiency of state-of-the-art statistical models and tools along-with the convenience and representational power of classical symbolic representations [5]. This permits the last three decades of research on graph based structural representations in various domains [1, to benefit from the state-of-the-art machine learning models and tools. Graph embedding has its application to the whole variety of domains that are entertained by pattern recognition and where the use of a relational data structure is mandatory for performing high level semantic tasks. Apart from reusing the computational efficient methods for vector spaces, another important motivation behind graph embedding methods is to solve the computationally hard problems geometrically [6. We refer the interested reader to [7] 8] for further reading on graph embedding.

The graph embedding methods are formally categorized as implicit graph embedding or explicit graph embedding. The implicit graph embedding methods are based on graph kernels. A graph kernel is a function that can be thought of as a dot product in some implicitly existing vector space. Instead of mapping graphs from graph space to vector space and then computing their dot product, the value of the kernel function is evaluated in graph space. Such an embedding satisfies the main mathematical properties of dot product. However, since it does not explicitly map a graph to a point in vector space, a strict limitation of implicit graph embedding is that it does not permit all the operations that could be defined on vector spaces. We refer the interested reader to [7] [9] for further reading on graph kernels and implicit graph embedding.

On the other hand, the more useful, explicit graph embedding methods explicitly embed an input graph into a feature vector and thus enable the use of all the methodologies and techniques devised for vector spaces.

Definition 1. Attributed graph (AG). Let $A_{V}$ and $A_{E}$ denote the domains of possible values for attributed vertices and edges respectively. These domains are assumed to include a special value that represents a null value of a vertex or an edge. An attributed graph $A G$ over $\left(A_{V}, A_{E}\right)$ is defined to be a four-tuple:

where,

$$
A G=\left(V, E, \mu^{V}, \mu^{E}\right)
$$

$V$ is a set of vertices,

$E \subseteq V \times V$ is a set of edges,

$\mu^{V}: V \longrightarrow A_{V}^{k}$ is function assigning $k$ attributes to vertices and $\mu^{E}: E \longrightarrow A_{E}^{l}$ is a function assigning $l$ attributes to edges.

Definition 2. Explicit graph embedding. Explicit graph embedding maps a graph to a point in suitable vector space. It encodes the graphs 
by equal size vectors and produces one vector per graph. Mathematically, for a graph $A G=\left(V, E, \mu^{V}, \mu^{E}\right)$, explicit graph embedding is a function $\phi$, which maps graph $A G$ from graph space $G$ to a point $\left(f_{1}, f_{2}, \ldots, f_{n}\right)$ in $n$ dimensional vector space $\mathbb{R}^{n}$ :

$$
\begin{aligned}
\phi: G & \longrightarrow \mathbb{R}^{n} \\
A G \longmapsto \phi(A G) & =\left(f_{1}, f_{2}, \ldots, f_{n}\right)
\end{aligned}
$$

The vectors obtained by an explicit graph embedding method can also be employed in a standard dot product for defining an implicit graph embedding function between two graphs [11. An interesting property of explicit graph embedding is that the graphs are embedded in pattern spaces in a manner that similar structures come close to each other and different structures goes far away i.e. an implicit clustering is achieved [12. Another important property of explicit graph embedding is that the graphs of different size and order need to be embedded into a fixed size feature vector. This means that for constructing the feature vector, an important step is to mark the important details that are available in all the graphs and are applicable to a broad range of graph types. We refer the interested reader to 7 for further reading on explicit graph embedding.

\section{$1.1 \quad$ Related Works}

Recently, two interesting series of works on explicit graph embedding for pattern recognition, with an application to graphics recognition, have been proposed in literature.

The first method is from Bunke et al. [11] and is based on dissimilarity of a graph from a set of prototypes. The main idea of this work is to construct a vector of graph edit distances from the graph to be embedded and a set of $k$ prototypes selected in the graph database. The embedding of the graph is thus a vector of $k$ distances. Formally, let $\Gamma=g_{1}, \ldots, g_{n}$ be a set of graphs and $p=p_{1}, \ldots, p_{k} \subset \Gamma$ be a subset of selected prototypes from $\Gamma$. The graph embedding is defined as the function $\Phi: \Gamma \longmapsto(\mathbb{R})^{k}$, such that $\Phi(g)=\left[d\left(g, p_{1}\right), \ldots, d\left(g, p_{k}\right)\right]$ where $d\left(g, p_{i}\right)$ is the graph edit distance between graph $g$ and the $i^{\text {th }}$ prototype graph in $p$. In [13, the authors propose an improvement of the graph embedding method by using feature selection methods. This type of projection is very interesting as it offers computational advantages over the traditional graph based algorithms. However, the limitation of setting the edit distance is found in this method. In addition, the choice of prototype graphs is also a significant parameter as it determines the size of the vector and its capacity to effectively represent the graph in the vector space. Also, it remains highly dependent on the application and its learning set.

The second method is from Gibert et al. 14] and is based on the frequencies of appearance of specific knowledge-dependent substructures in graph. The main idea of this work is to construct vector representation of graphs by counting the frequency of appearance of specific set of representatives of node labels and their corresponding edges. In [15] the authors propose an improvement of their 
graph embedding technique by dimensionality reduction of the obtained feature vector. In [16] the authors have applied multiple classifiers to their graph embedding method. In [14 the authors have studied the application of feature selection algorithms for their graph embedding method. This type of graph embedding algorithms provides an embedding of graph into feature vector, in linear time complexity. Their simplicity of implementation is an important advantage. However, the features that have been used, are very localized to nodes and arcs. The graph embedding contains little information on the topology, which can have a negative impact on the classification results.

\subsection{Main Contribution of This Paper}

This paper is a continuation of our work on explicit graph embedding. The method is originally proposed in [17] 18] and is named as "fuzzy multilevel graph embedding - FMGE". FMGE embeds a graph into feature vector space by extracting a large number of features from graph. The use of high dimensional feature vector permits FMGE to achieve generalization to diverse graphs in an unsupervised fashion. However this also results into high dimensionality and sparsity of feature vector. In [19] we studied the application of dimensionality reduction techniques on FMGE extracted features. Motivated from the similar works in [13] and [14] where the authors have applied feature selection algorithms on explicit graph embedding methods, in this paper we take forward our work on graph embedding and study the application of feature selection algorithms on FMGE extracted features.

The rest of this paper is organized as follows. In Section 2 we briefly outline the Fuzzy Multilevel Graph Embedding (FMGE). In Section 3 we describe the application of feature selection algorithms on FMGE. Experimentation and discussion is presented in Section 4 . In Section 5 we conclude this paper with future lines of research.

\section{Fuzzy Multilevel Graph Embedding (FMGE)}

Most of the existing works on graph embedding deal only the graphs that are comprised of edges with a single attribute and vertices with either no or only symbolic attributes. These methods are only useful for specific application domains for which they are designed. FMGE does not require any dissimilarity measure between graphs and to the best of our knowledge, FMGE extends the methods in literature by offering the embedding of attributed graphs with many numeric as well as symbolic attributes on both nodes and edges. It is applicable to directed as well as undirected attributed graphs. The time complexity of FMGE is linear to number of attributes and size of the graphs [18. Many existing solutions for graph embedding offer to utilize the statistical significant details in graphs for embedding them into feature vectors. FMGE exploits the topological, structural and attribute information of the graphs along-with the statistical significant information, for constructing feature vectors of adapted and 
optimal size. It employs fuzzy overlapping trapezoidal intervals for minimizing the information loss while mapping from continuous graph space to discrete feature vector space. The proposed feature vector is very significant for application domains where the use of graphs is mandatory for representing rich structural and topological information, and an approximate but computational efficient solution is needed. The unsupervised learning abilities of FMGE and the fact that it does not require a labeled graph dataset for learning allows its inexpensive deployment to various application domains [18. FMGE performs multilevel analysis of graph to extract discriminatory information of three different levels. These include the graph level information, structural level information and the elementary level information (see Fig. 1). The three levels of information represent three different views of graph for extracting global details, details on topology of graph and details on elementary building units of graph. The feature vector of FMGE is named Fuzzy Structural Multilevel Feature Vector - FSMFV (see Fig. 2).

The features for graph level information represent a coarse view of graph and give general information about the graph. These features include graph order and graph size.

The features for structural level information represent a deeper view of graph and are extracted from the node degrees and subgraph homogeneity in graph. Subgraph homogeneity is represented by computing resemblance attributes for the nodes and edges of graph. The resemblance attributes for an edge is computed from the attributes on its neighboring nodes. The resemblance for a numeric attribute $(a)$ is computed as a ratio of this attribute's values on neighboring nodes of an edge $\left(a_{1}\right.$ and $\left.a_{2}\right)$ (see Eq. 1). Whereas the resemblance for a symbolic attribute $(b)$ is computed as a ratio of this attribute's values on neighboring nodes of an edge $\left(b_{1}\right.$ and $\left.b_{2}\right)$ (see Eq. 2).

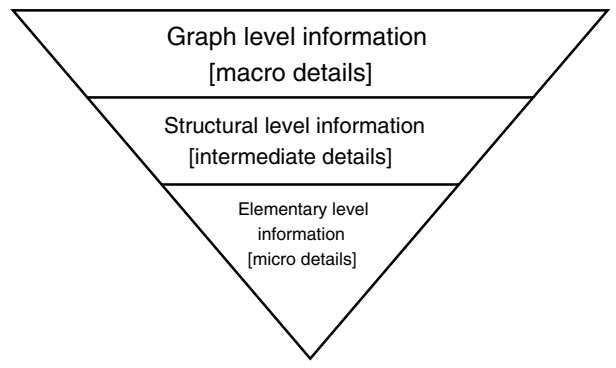

Fig. 1. Multi-facet view of discriminatory information in graph

\begin{tabular}{|c|c|c|c|c|c|}
\hline $\begin{array}{c}\text { Graph } \\
\text { order }\end{array}$ & $\begin{array}{c}\text { Graph } \\
\text { size }\end{array}$ & $\begin{array}{c}\text { Embedding of } \\
\text { node degree }\end{array}$ & $\begin{array}{c}\text { Embedding(s) of } \\
\text { subgraph(s) homogenity }\end{array}$ & $\begin{array}{c}\text { Embedding(s) of } \\
\text { node attribute(s) }\end{array}$ & $\begin{array}{c}\text { Embedding(s) of } \\
\text { edge attribute(s) }\end{array}$ \\
\hline
\end{tabular}

Fig. 2. Feature vector of FMGE 


$$
\begin{gathered}
\text { resemblance }\left(a_{1}, a_{2}\right)=\min \left(\left|a_{1}\right|,\left|a_{2}\right|\right) / \max \left(\left|a_{1}\right|,\left|a_{2}\right|\right) \\
\operatorname{resemblance}\left(b_{1}, b_{2}\right)=\left|\begin{array}{cc}
1 & b_{1}=b_{2} \\
0 & \text { otherwise }
\end{array}\right|
\end{gathered}
$$

The third level of information is extracted by penetrating into further depth and more granular view of graph and employing details of the elementary building blocks of graph. These features represent the information extracted from the node and edge attributes.

The node degree, numeric resemblance attributes, numeric node attributes and numeric edge attributes are embedded by fuzzy histograms whereas the symbolic resemblance attributes, symbolic node attributes and the symbolic edge attributes are embedded by crisp histograms. FMGE learns the intervals for constructing these histograms, during an unsupervised learning phase and employs these parameters during graph embedding phase [17] 18] [19].

Length of Feature Vector (FSMFV): The length of the feature vector is strictly dependent on the size of histograms used for encoding the three levels of information. The length of feature vector is uniform for all graphs in an input collection and is given by Eq, 3 .

where,

$$
\text { Length of FSMFV }=2+\sum s_{i}+\sum c_{j}
$$

- 2 refers to the features for graph order and graph size.

- $s_{i}$ refers to the number of bins in fuzzy interval encoded histogram for each numeric attribute $i$, in graph.

- $c_{j}$ refers to the number of bins in crisp interval encoded histogram for each symbolic attribute $j$, in graph.

\section{Feature Selection by Ranking Discriminatory Features}

The feature vector obtained by FMGE is based on histogram encoding of the multilevel information extracted from graph. The number of features in the vector is directly dependent on the number of bins employed for constructing these histograms. The use of high dimensional histograms is explicitly built into the method as it enables FMGE to provide a more robust encoding of information and enables it to generalize to unseen graphs. However, this results into a serious drawback as well, that the feature vector becomes sparse and confuses between classes of graphs. Previously we have tried to reduce the dimensionality of the feature vector by using principal component analysis (PCA) [19]. PCA is based on linear transformation of data to a low dimensional space that describes most of variance in data. But we feel that instead of an unsupervised dimensionality reduction technique like $\mathrm{PCA}$, the use of a supervised dimensionality reduction technique (a.k.a. feature selection) will result into a more meaningful ranking of the FMGE extracted features. This ranking will permit to select the high 
discriminatory features and ignore the confusing features in the FMGE embedded vector space. Thus producing more compact feature vector representation of graphs and adding strength to the discriminatory power of the feature vector.

We have employed the Relief algorithm based feature selection [20. The Relief algorithm is a classical ranking method that is based on the ability of features to discriminate between different classes. For each instance of a given feature, the near-hit (closest value among elements of same class) and the near-miss (closest value among element of other classes) are computed. A weight is calculated for every feature in terms of the distances of each sample to its near-hit and nearmiss. Mathematically, for a set $S$ of $m$ samples of feature $f_{i}$, the rank value $\omega_{f_{i}}$ is computed as:

$$
\omega_{f_{i}}=\frac{1}{m} \sum_{x \in S}\left|x-Z_{x}^{-}\right|-\left|x-Z_{x}^{+}\right|
$$

where,

- $Z_{x}^{-}$is near-miss of sample $x$.

- $Z_{x}^{+}$is near-hit of sample $x$.

A high ranking value of $\omega_{f_{i}}$ is desirable as it indicates that the feature is important and has high discriminatory capabilities. In order to reduce the size of FMGE feature vector and to remove the unimportant features from it, we select the subset of top-ranked features, on the basis of ranks obtained through the Relief algorithm.

\section{Experimentation}

The experimentation has been performed on 'IAM Graph Database Repository for Graph Based Pattern Recognition and Machine Learning'. The IAM graph database repository is publicly available from the website of IAPR technical committee on graph based representations (TC-15) 1 , and contains graph datasets from the field of document image analysis and graphics recognition, describing both synthetic and real data [21].

\subsection{Datasets}

The summary of the letter, GREC and fingerprint datasets, together with some characteristic properties, is given in Table 1. The letter graph dataset is comprised of graphs extracted from drawings of 15 capital letters of Roman alphabet that consists of straight lines only. The prototype drawing of letters are converted into prototype graphs by representing lines by undirected edges and ending points of lines by nodes. Each node is labeled with a two-dimensional attribute giving its position relative to a reference coordinate system. The GREC graph dataset is comprised of graphs representing 22 symbols from architectural and electronic drawings. Graphs are extracted from the denoised images

\footnotetext{
1 http://www .greyc.ensicaen.fr/iapr-tc15/index.php
} 
Table 1. IAM graph database

\begin{tabular}{|c|c|c|c|c|}
\hline \multicolumn{2}{|c|}{} & Letter LOW & GREC & Fingerprint \\
\hline Size & Train & 750 & 836 & 500 \\
& Valid & 750 & 836 & 300 \\
& Test & 750 & 1628 & 2000 \\
\hline \multicolumn{2}{|c|}{ Classes } & 15 & 22 & 4 \\
\hline Average & $|V|$ & 4.7 & 11.5 & 5.4 \\
& $|E|$ & 3.1 & 12.2 & 4.4 \\
\hline Maximum & $|V|$ & 8 & 25 & 26 \\
& $|E|$ & 6 & 30 & 25 \\
\hline Numeric attribute & $|V|$ & 2 & 2 & 2 \\
& $|E|$ & 0 & 1 & 1 \\
\hline Symbolic attribute & $|V|$ & 0 & 1 & 0 \\
& $|E|$ & 0 & 1 & 0 \\
\hline
\end{tabular}

by representing ending points, corners, intersections and circles by nodes and labeled with a two-dimensional attribute giving their position. The nodes are connected by undirected edges that are labeled as line or arc and have the angle with respect to the horizontal direction as attribute. Fingerprint images are converted into graphs by representing the ending points and bifurcation points of the skeletonized regions as nodes. Each node is labeled with a two-dimensional attribute giving its position. The edges are attributed with an angle denoting the orientation of the edge with respect to the horizontal direction.

\subsection{Experimental Setup and Results}

We have evaluated the application of Relief feature selection algorithm on FMGE, by classification rate obtained by a nearest neighbor classifier. The experiments are performed by first tuning the parameters on the validation set and then using the best configuration on the test set.

The first validation parameter is the number of fuzzy intervals for embedding numeric information in graph i.e. the node degree, numeric resemblance attributes, numeric node attributes and numeric edge attributes. Starting from 2 intervals, the number of fuzzy intervals for embedding the numeric information is increased until 25 (in steps of 1).

The second validation parameter is selection of top-ranked features. For each of the 25 configurations of FMGE, we applied the Relief feature selection algorithm (with a neighborhood size of 10 for calculating near-hit and near-miss), to obtain rankings of features. We used this ranking information to generate all subsets of high to low ranked features (for each of the 25 configuration of FMGE), i.e. subset containing top-1 feature, subset containing top-2 feature, subset containing top-3 feature and so on. We validated the classification rate for all of these subsets of features and selected the subset of features that produced the best classification rate on validation set (for each of the 25 configurations of FMGE). Fig. 3 shows the validation results for letter LOW, GREC and Fingerprint datasets. The colored curves in the plot represent the first validation parameter i.e. number of the fuzzy intervals for encoding numeric information. Whereas, each point on a curve gives the classification rate obtained on the $n$ top-ranked features. The plots clearly demonstrate that the method can obtain its maximum classification rates on only a small subset of top-ranked features. 


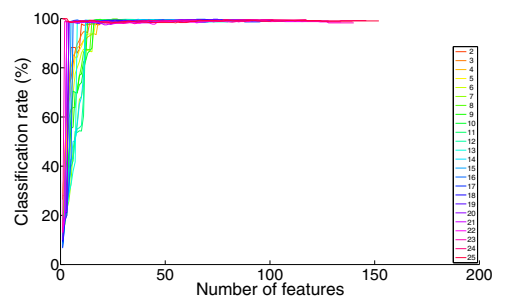

(a) letter LOW

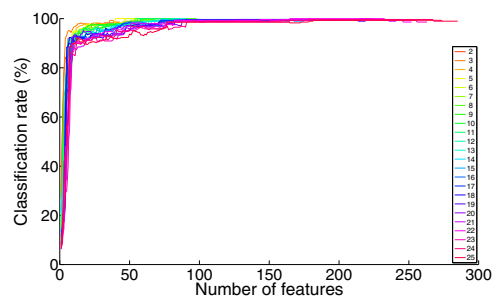

(b) GREC

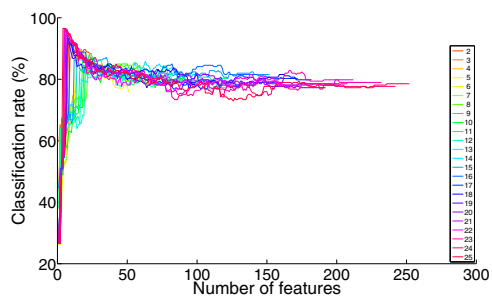

(c) Fingerprint

Fig. 3. Validation results showing the classification rates obtained for different subsets of top-ranked features for the 25 configurations of FMGE. The horizontal axis contains sorted (high to low ranked by Relief algorithm) features extracted by FMGE.

Table 2. Results on test sets - IAM graph database. CR is the classification rate (\%) obtained by $k-n n$ classifier and DIM is the dimensionality of feature vector.

\begin{tabular}{|l|c|c|c|c|c|c|}
\hline & \multicolumn{2}{|l|}{ Letter LOW } & \multicolumn{2}{|c|}{ GREC } & \multicolumn{2}{|c|}{ Fingerprint } \\
\cline { 2 - 7 } & CR & DIM & CR & DIM & CR & DIM \\
\hline Reference system [21 & 99.6 & & 95.5 & & 76.6 & \\
\hline Gibert et al. [14] (best CR) & $\mathbf{1 0 0}$ & & 98.7 & & 80.5 & \\
\hline Gibert et al. [4] (Relief feature selection) & 99.1 & & 96.4 & & 77.6 & \\
\hline \hline Full FMGE vector & 96.5 & 58 & 97.2 & 79 & 76.6 & 127 \\
\hline FMGE vector (PCA reduced [19]) & 96.3 & 10 & 96.8 & 14 & 77.2 & 16 \\
\hline \hline FMGE vector (Relief feature selection) & 99.2 & 37 & $\mathbf{9 9 . 8}$ & 47 & $\mathbf{8 5 . 5}$ & 61 \\
\hline
\end{tabular}

The configuration of FMGE with optimized subset of top-ranked features (w.r.t. classification rate on validation set), was used to embed the test set and to compute the final classification rates on test set.

Table 2 presents the classification rates of a $k$ - $n n$ classifier in FMGE embedded vector space on test set, before and after the application of feature selection technique. The PCA dimensionality reduction results are reported from [19. For comparison of results, the $k$ - $n n$ classification rates are reported for the graph edit distance plus $k$ - $n n$ classifier based reference system 21] and $k$-nn classifier based system of Gibert et al. [14.

The results show that the application of feature selection technique successfully improves the performance of FMGE representation of graphs. The Relief feature selection technique selects much lower number of features (as compared to full FMGE vector) and in all cases improves the classification rates obtained 
by $k$-nn classifier in FMGE embedded vector spaces. Although the vectors obtained after feature selection have slightly higher dimensionality than PCA reduced vectors. However, feature selection technique enables FMGE to obtain better classification rates than those of PCA reduced vectors. The feature selection technique also provides a deep insight into the groups of features that are discriminatory and the features that are not very useful for a graph dataset. The application of feature selection algorithm on FMGE vectors permits to improve the quality of FMGE embedding and eventually the performance of FMGE. During classification step, as a result of lower dimensionality of the feature vector the computation time of classifier was reduced as well.

\section{Conclusion}

In this paper we have studied the application of feature selection algorithms to our earlier proposed unsupervised learning based explicit graph embedding method (the Fuzzy Multilevel Graph Embedding - FMGE). The use of a feature selection technique on FMGE extracted features enables to obtain a ranking of features, for selecting the top-ranked features and discarding the unimportant features. This permits to increase the quality of embedding and improves the performance of FMGE. Feature selection step also improves the unsupervised learning capabilities of FMGE by adapting its feature-set to underlying graph dataset. The initial experimental results are very encouraging and in future we plan to study the application of sophisticated feature selection techniques for further improving the quality of Fuzzy Multilevel Graph Embedding.

\section{References}

1. Conte, D., Foggia, P., Sansone, C., Vento, M.: Thirty years of graph matching in pattern recognition. International Journal of Pattern Recognition and Artificial Intelligence 18(3), 265-298 (2004)

2. Bunke, H., Irniger, C., Neuhaus, M.: Graph Matching - Challenges and Potential Solutions. In: Roli, F., Vitulano, S. (eds.) ICIAP 2005. LNCS, vol. 3617, pp. 1-10. Springer, Heidelberg (2005)

3. Bunke, H., Gunter, S., Jiang, X.: Towards Bridging the Gap between Statistical and Structural Pattern Recognition: Two New Concepts in Graph Matching. In: Singh, S., Murshed, N., Kropatsch, W.G. (eds.) ICAPR 2001. LNCS, vol. 2013, pp. 1-11. Springer, Heidelberg (2001)

4. Roth, V., Laub, J., Kawanabe, M., Buhmann, J.: Optimal cluster preserving embedding of nonmetric proximity data. IEEE Transactions on Pattern Analysis and Machine Intelligence 25(12), 1540-1551 (2003)

5. Chen, T., Yang, Q., Tang, X.: Directed graph embedding. In: International Joint Conference on Artificial Intelligence, pp. 2707-2712 (2007)

6. Shaw, B., Jebara, T.: Structure preserving embedding. In: International Conference on Machine Learning, pp. 1-8 (2009)

7. Foggia, P., Vento, M.: Graph Embedding for Pattern Recognition. In: Ünay, D., Çataltepe, Z., Aksoy, S. (eds.) ICPR 2010. LNCS, vol. 6388, pp. 75-82. Springer, Heidelberg (2010) 
8. Lee, G., Madabhushi, A.: Semi-Supervised Graph Embedding Scheme with Active Learning (SSGEAL): Classifying High Dimensional Biomedical Data. In: Dijkstra, T.M.H., Tsivtsivadze, E., Marchiori, E., Heskes, T. (eds.) PRIB 2010. LNCS, vol. 6282, pp. 207-218. Springer, Heidelberg (2010)

9. Riesen, K., Bunke, H.: Graph Classification and Clustering Based on Vector Space Embedding. World Scientific (2010)

10. Riesen, K., Bunke, H.: Graph Classification And Clustering Based On Vector Space Embedding. World Scientific Publishing Co., Inc. (2010)

11. Bunke, H., Riesen, K.: Recent advances in graph-based pattern recognition with applications in document analysis. Pattern Recognition 44(5), 1057-1067 (2011)

12. Wilson, R.C., Hancock, E.R., Luo, B.: Pattern vectors from algebraic graph theory. IEEE Transactions on Pattern Analysis and Machine Intelligence 27(7), 1112-1124 (2005)

13. Bunke, H., Riesen, K.: Improving vector space embedding of graphs through feature selection algorithms. Pattern Recognition 44(9), 1928-1940 (2011)

14. Gibert, J., Valveny, E., Bunke, H.: Feature selection on node statistics based embedding of graphs. Pattern Recognition Letters (April 2012)

15. Gibert, J., Valveny, E., Bunke, H.: Dimensionality Reduction for Graph of Words Embedding. In: Jiang, X., Ferrer, M., Torsello, A. (eds.) GbRPR 2011. LNCS, vol. 6658, pp. 22-31. Springer, Heidelberg (2011)

16. Gibert, J., Valveny, E., Terrades, O.: Multiple classifiers for graph of words embedding. Multiple Classifier Systems, 1-10 (2011)

17. Luqman, M.M., Lladós, J., Ramel, J.Y., Brouard, T.: A Fuzzy-Interval Based Approach for Explicit Graph Embedding. In: Ünay, D., Çataltepe, Z., Aksoy, S. (eds.) ICPR 2010. LNCS, vol. 6388, pp. 93-98. Springer, Heidelberg (2010)

18. Luqman, M.M., Ramel, J.Y., Lladós, J., Brouard, T.: Fuzzy Multilevel Graph Embedding. Pattern Recognition (accepted, 2012), http://dx.doi.org/10.1016/j.patcog.2012.07.029

19. Luqman, M.M., Lladós, J., Ramel, J.Y., Brouard, T.: Dimensionality Reduction for Fuzzy-Interval Based Explicit Graph Embedding. In: GREC, pp. 117-120 (2011)

20. Kira, K., Rendell, L.A.: The feature selection problem: traditional methods and a new algorithm. In: Proceedings of the Tenth National Conference on Artificial Intelligence, AAAI 1992, pp. 129-134. AAAI Press (1992)

21. Riesen, K., Bunke, H.: IAM Graph Database Repository for Graph Based Pattern Recognition and Machine Learning. In: da Vitoria Lobo, N., Kasparis, T., Roli, F., Kwok, J.T., Georgiopoulos, M., Anagnostopoulos, G.C., Loog, M. (eds.) S+SSPR 2008. LNCS, vol. 5342, pp. 287-297. Springer, Heidelberg (2008) 